\title{
ORDERED POWER ASSOCIATIVE GROUPOIDS
}

\author{
DESMOND A. ROBBIE
}

\begin{abstract}
Compact, connected, totally ordered, (Hausdorff) topological groupoids, with restrictions on their sets of idempotents and with varying degrees of power associativity assumed, are examined. The paper evolves from the author's example of such a groupoid which has only two idempotents (a zero for least element, and an identity for greatest element), a compact neighborhood of the greatest element consisting of power associative elements, and which is not isomorphic to either the real thread or the nil thread. Another example given has a zero for least element, an idempotent for greatest element, and no other idempotents, and has a compact neighborhood of the greatest element consisting of an associative subgroupoid in which all products are equal to the greatest element. Theorems are given which show that these examples, and one other, in some sense, exhaust the possibilities.
\end{abstract}

Ordered power associated groupoids have been considered by-among many others-J. Aczel [1], L. Fuchs [2], K. H. Hofmann [4], P. S. Mostert [7], and R. J. Warne [10]. We shall provide an example which complements some work of Mostert, and a result which improves somewhat a result given by Warne. Thanks are due to Professors Hofmann and Mostert who read the original draft and made detailed suggestions for improvement, and also to the referee who suggested major improvements. Due to their help we are able to give a more complete account than originally envisaged.

The direction of Professor A. D. Wallace during this research is gratefully acknowledged.

A groupoid is a nonvoid Hausdorff space together with a continuous binary operation (multiplication denoted by juxtaposition). An ordered groupoid is the data of an ordered set together with the order topology, in which the multiplication is continuous. An element of a groupoid is power associative if it is contained in an associative subgroupoid. A sect is an ordered groupoid which is compact and connected (of course in the order topology), which has a zero for least element $(0 x=x 0=0$ whatever $x$ ), and an idempotent, $e$, for the greatest element (with $0<e$ ).

Presented to the Society, January 22, 1971; received by the editors October 1, 1970. AMS 1969 subject classifications. Primary 2205; Secondary 2095.

Key words and phrases. Ordered groupoid, power associative, sect, cancellative, real thread, nil thread, isomorphism. 
(Familiarity with the structure of ordered spaces is assumed, for example, R. L. Wilder [11], or Hocking and Young [3].) An ordered groupoid is termed cancellative if each element except the least is known to cancel on each side.

For reference below we note two well-known sects which are, however, quite special in that in each case the multiplication is commutative and associative. The first is the real interval $[0,1]$ with ordinary real multiplication which is often termed the real thread, and which is a cancellative sect. The second example, often called the nil thread, is the real interval $\left[\frac{1}{2}, 1\right]$ with the multiplication $x \circ y=\max \left\{\frac{1}{2}, x y\right\}$ where $x y$ is ordinary real multiplication. Two groupoids are isomorphic if there is a homeomorphism of one onto the other which is also a morphism.

We begin our analysis with three examples.

ExAmple 1. Take $T=[0,1]$, the real interval, and define

$$
\begin{array}{rlrl}
x \circ y & =2 y, & & \left\{(x, y): 0 \leqq x \leqq \frac{1}{2} \text { and } 0 \leqq y \leqq x\right\} \\
\cup\left\{(x, y): \frac{1}{2} \leqq x \leqq 1 \text { and } 0 \leqq y \leqq 1-x\right\}, \\
& =1-x+y, & & \left\{(x, y): \frac{1}{2} \leqq x \leqq 1 \text { and } 1-x \leqq y \leqq x\right\}, \\
& =y \circ x, & & \text { otherwise. }
\end{array}
$$

We claim that this example is a sect which has an identity for the greatest element, no other idempotents, and has a neighborhood of the identity containing only power associative elements. Also, it is clearly not isomorphic to the real thread or the nil thread. The establishment of these claims is left to the reader but we note that the induction part of the argument, while quite easy, is a little unusual. Each element $x$ which is in the interval $\left[\frac{1}{2}, 1\right]$ is power associative and this is proved by using that, for such elements, $x \circ x=1$.

EXAmple 2. Take the usual real interval $[0,1]$ with the operation of geometric mean.

We claim that this is a sect in which all elements are power associative, in fact idempotent, which is cancellative, commutative, and which satisfies the condition of mediality $((a b)(c d)=(a c)(b d))$. We are indebted to Professor K. N. Sigmon for pointing out that this well-known sect has all these properties since at first we had a much more complicated looking example: (Of course all such sects are isomorphic.) (See [8] for our original example.)

These examples serve to illustrate that in a sect we need more than a neighborhood of power associative elements about the maximal idempotent to cut down pathology, and that on the other hand we need a restriction on the set of idempotents. 
We give our remaining example at this point.

EXAMPLE 3. Take $T=[0,1]$, the usual real interval, and define

$$
\begin{aligned}
x \circ y & =2 y, & & \left\{(x, y): 0 \leqq x \leqq 1 \text { and } 0 \leqq y \leqq \min \left\{x, \frac{1}{2}\right\}\right\}, \\
& =1, & & \left\{(x, y): \frac{1}{2} \leqq x \leqq 1 \text { and } \frac{1}{2} \leqq y \leqq x\right\}, \\
& =y \circ x, & & \text { otherwise. }
\end{aligned}
$$

It is clear that $T$ is a sect with only two idempotents and that the set of elements greater than or equal to $\frac{1}{2}$ and less than or equal to 1 form an associative subgroupoid in which the product of any two elements is 1 . This example is important in relation to Theorem 2 below.

Before giving the next result we remind the reader that we do not assume that multiplication preserves order. The result was suggested to us by Professor K. H. Hofmann after looking at Examples 1 and 2 above and Corollary 1.2 below. He also gave much of the proof although we had also progressed along the same path.

THEOREM 1 [K. H. H.]. Given a sect $T$ in which all elements are power associative, then, for each element $t$ in $T$, there are the following possibilities:

(a) $t$ is an idempotent, or

(b) $t$ is an interior point of a closed interval semigroup which is isomorphic to either the real thread or the nil thread.

Proof. Since the continuous image of a connected set is connected, and all power maps take the least element 0 to 0 and the greatest element $e$ to $e$, and since the only connected sets are intervals, we have that all power maps take the sect onto itself. Thus, the sect is divisible. Now we do not know that roots are unique but we do know that the set of $n$th roots of an element $x$, for a fixed natural number $n$, is a closed set, and so has a least element. We denote this least element by $x^{1 / n}$.

It is tedious but easy to show (see Robbie [8]) that if we define $t^{p / q}$ to be $\left(t^{1 / q}\right)^{p}$ for positive integers $p$ and $q$, then the map $f: Q^{+} \rightarrow T$, given by $p / q \mapsto t^{p / q}$, is a well-defined algebraic morphism, and, of course, $f(1)=t$. The proof requires again the use of the zero and the order properties, and connectivity properties of our space.

For the next step let

$$
C=\bigcap\left\{f((0, r])^{*}: 0<r\right\} .
$$

Then $C$ is the intersection of a tower of nonempty compact sets and so is nonempty. Moreover, each of the sets, being the closure of a commutative and associative set, will be commutative and associative as well, and so then $C$ will have these properties also. We note next that by juggling backwards and forwards with the morphism $f$ we can show that $C C \subset C$ so that $C$ is a compact abelian semigroup. Again by using a similar 
procedure it can be shown that $C C=C$ and that $C$ has only one idempotent. Then, by a result of Koch and Wallace [6], $C$ is a group. Still again it is easily seen that $C$ is divisible and so must be a connected group. Since a topological group is homogeneous in its topology, $C$ must be a singleton. Denoting this single element by $c$ we have, of course, that $c^{2}=c$. Then $f$ may be extended to $Q^{+} \cup\{0\}$ by $f(0)=c$ and this extension is continuous at each point (see Hofmann [4, p. 21]). Next, considering $f$ as a function from $Q^{+} \cup\{0\}$ into $(\operatorname{im} f)^{*}$, since $(\operatorname{im} f)^{*}$ is a compact abelian semigroup, it is well known that $f$ may be extended continuously to a morphism from all nonnegative reals into $(\operatorname{im} f)^{*}$. Thus, $(\operatorname{im} f)^{*}$ is a solenoidal subsemigroup of a totally ordered semigroup and so is isomorphic to the real thread, the nil thread, or a point.

COROLlary 1.1. With the hypotheses of Theorem 1 and the additional hypothesis that there are only two idempotents, then $T$ must be isomorphic to either the real thread, or the nil thread.

Proof. Immediate.

COROLlaRY 1.2. If $T$ is a cancellative sect in which all elements are power associative and in which the maximal idempotent, $e$, is isolated in the set of idempotents, then $T$ is isomorphic to the real thread.

Proof. (See Robbie [8] for a direct proof by a simple order argument.) $T$, by Theorem 1 and the cancellative property, must contain a nondegenerate semigroup isomorphic to the real thread, one end of which is the maximal idempotent $e$. Now $e$ cannot be the zero for that semigroup (again due to the cancellation property). Finally, again by the cancellation property, the zero for this semigroup must be the zero of $T$.

The referee suggested another theorem which after some further modification became the next result. It should be noted also that Mostert [7], produced similar working to that necessary to prove part of the theorem, so we have omitted most of the details for that and concentrated on the newer part. Professor Hofmann had earlier suggested that a similar result might be true.

THEOREM 2 [REFEREE AND AUTHOR]. If $T$ is a sect with just two idempotents, and, if there is a neighborhood of the maximal idempotent $e$ consisting of power associative elements, then,

(a) either $e \cdot V=V \cdot e=e$ for some neighborhood of $e$, or $e$ is an identity over some neighborhood of $e$, and

(b) either $e$ is a cluster point of elements $t$ with $(t e)^{2}=e$, or $T$ is the real thread, or the nil thread. 
Proof. It is an elementary exercise in the connectedness properties of ordered spaces that either all elements, $t$, strictly between 0 and $e$ have the property $t^{2}<t$, or they all have the property $t^{2}>t$. In case $t^{2}<t$, consider a net $\left\{t_{\alpha}\right\}$ of power associative elements converging monotonically to $e$. (This can be obtained if $e$ is merely a cluster point of power associative elements, but the extra hypothesis is needed in the case $t^{2}>t$ to get our result.) Choose $0<a<e$. Now 0 must be the idempotent in the closure of the set of powers of $T$ for each $t$ in the net. Thus for each $\alpha$, there is an integer $n(\alpha)$ such that $x^{k} \geqq a$, for $1 \leqq k \leqq n(\alpha)$, but $x^{n(\alpha)+1}<a$. Then by taking the index set of the net with the discrete topology, and choosing a particular point, $q$, of the Stone-Cech compactification of the net, we may define a one parameter semigroup in our groupoid by $\sigma(s)=f_{s}(q)$, where $f_{s}(\cdot)$ is the continuous extension to the Stone-Cech compactification of the net, of the function defined on the net by $f_{s}(\alpha)=x^{[s n(\alpha)]}$, where $[s n(\alpha)]$ denotes the greatest integer function. The details of this type of argument are well known (see Hofmann and Mostert [5]). We remark that once this is established then the proof goes again similarly to that outlined in the proof of Theorem 1 . We note here that the Koch and Wallace result mentioned earlier seems fundamental for these types of arguments. We end up then, in this case, with either the real thread or the nil thread.

If now we assume that $t^{2}>t$ for all $t$, then, for any power associative $t$, the idempotent in the closure of the powers must be $e$. Then also $e$ associates with $t$ and so $e$ is the identity for $t e$. Now te must then be power associative and so since $(t e)^{2^{m}}$ approaches $e$, then we obtain that the closure of the set of powers of $t e$ is either a group of order $2^{m}$ for some $m$ or it is a 2-adic group. (The topology of a topological group is homogeneous.) If there are not elements, $t$, arbitrarily close to $e$ with the property $(t e)^{2}=e$, but there is a neighborhood about $e$ with only power associative elements, then, choosing $t<e$ sufficiently close to $e$ so that $t e$ is also power associative, we cannot have $(t e)^{3}=t e$, since this would give $\left((t e)^{2}\right)^{2}=(t e)^{2}$ which in turn would mean $(t e)^{2}=e$, a contradiction. So either $(t e)^{3}>t e$ for all $t<e$ in that neighborhood, or $(t e)^{3}<t e$ for all those $t$. The second case is not possible since it would lead to the conclusion that 0 belongs to the closure of the set of powers of $t e$, but this set is included in the closure of the set of powers of $t$, because $e$ belongs to the latter set which is a semigroup. So always if $t(<e)$ is in a certain neighborhood of $e$, then $(t e)^{3}>t e$. By induction, it may be shown that $(t e)^{n}>$ $t e$ for each $n$ and so this means that te is the least element of the group about $t e$ which we are discussing. Then, due to the nature of the group, it is uniquely three divisible and so choosing the cube root, $r$, of te, we 
have by connectivity that $r=g e$ for some $t \leqq g\left\langle e\right.$. Then $\left.(g e)^{3}\right\rangle$ $g e \geqq t e$, which is a contradiction. Thus if $t^{2}>t$ for all elements between 0 and $e$, and there is a neighborhood of $e$ consisting of power associative elements, then $e$ is a cluster point for elements, $t$, with the property $(t e)^{2}=e$.

Finally we note that the next result does not seem readily to admit to an approach directly similar to those given above but comes from a more involved version of Warne's [10] work combined with work similar to that of Aczel [1]. The proof is omitted as it can be obtained from the author's dissertation [8]. We note however that Professor K. N. Sigmon has suggested the possibility of obtaining it from Corollary 1.2 by applying a direct limit type of argument similar to that used in his paper [9].

THEOREM 3. If $T$ is a cancellative compact connected ordered power associative groupoid (all elements power associative), with a zero for the least element, and a nonidempotent for the greatest element, and if as well the squaring function is a morphism, then $T$ is isomorphic to $\left[0, \frac{1}{2}\right]$ under ordinary real multiplication.

\section{BIBLIOGRAPHY}

1. J. Aczél, Quasigroups, nets and nomograms, Advances in Math. 1 (1965), 383-450. MR 33 \#1395.

2. L. Fuchs, Partially ordered algebraic systems, Pergamon Press, New York; Addison-Wesley, Reading, Mass., 1963, pp. 181-188. MR 30 \#2090.

3. J. G. Hocking and G. S. Young, Topology, Addison-Wesley, Reading, Mass., 1961. MR 23 \#A2857.

4. K. H. Hofmann, Zur mathematischen Theorie des Messens, Rozprawy Mat. 32 (1963), 32 pp. MR 33 \#3275.

5. K. H. Hofmann and P. S. Mostert, Elements of compact semigroups, Merrill, Columbus, Ohio, 1966. MR 35 \#285.

6. R. J. Koch and A. D. Wallace, Maximal ideals in compact semigroups, Duke Math. J. 21 (1954), 681-685. MR 16, 112.

7. P. S. Mostert, Comments on a paper of Warne, Mimeographed sheets, Tulane University, New Orleans, La., 1963.

8. D. A. Robbie, Some theorems on binary topological algebras, Dissertation, University of Florida, Gainesville, Fla., 1970.

9. K. N. Sigmon, Cancellative medial means are arithmetic, Duke Math. J. 38 (1970), 143-146.

10. R. J. Warne, Connected ordered topological groupoids with idempotent endpoints, Publ. Math. Debrecen 8 (1961), 143-146. MR 24 \#A538.

11. R. L. Wilder, Topology of manifolds, Amer. Math. Soc. Colloq. Publ., vol. 32, Amer. Math. Soc., Providence, R.I., 1949. MR 10, 614.

Department of Mathematics, University of Florida, Gainesville, Florida 32601

Department of Mathematics, Western Illinois University, Macomb, Illinois 61455 